\title{
Transcatheter closure of paravalvular leaks using a paravalvular leak device - a prospective Polish registry
}

\author{
Grzegorz Smolka ${ }^{1}$, Piotr Pysz ${ }^{2}$, Michał Kozłowski ${ }^{1}$, Marek Jasiński³, Radosław Gocoł ${ }^{3}$, Tomasz Roleder², \\ Agnieszka Kargul ${ }^{4}$, Andrzej Ochała ${ }^{1}$, Wojciech Wojakowski ${ }^{1}$ \\ ${ }^{1} 3^{\text {rd }}$ Division of Cardiology, Medical University of Silesia, Katowice, Poland \\ ${ }^{2} 2^{\text {nd }}$ Division of Cardiology, Medical University of Silesia, Katowice, Poland \\ ${ }^{3}$ Division of Cardiac Surgery, Medical University of Silesia, Katowice, Poland \\ ${ }^{4} 2^{\text {nd }}$ Department of Physiological and Medical Sciences, The Jerzy Kukuczka Academy of Physical Education, Katowice, Poland
}

Adv Interv Cardiol 2016; 12, 2 (44): 128-134

DOI: 10.5114 /aic.2016.59363

\section{Abstract}

Introduction: Transcatheter paravalvular leak closure (TPVLC) has become an established treatment option but is mostly performed with off-label use of different non-dedicated occluders. The first one specifically designed for TPVLC is the paravalvular leak device (PLD - Occlutech).

Aim: We present initial short-term results of a prospective registry intended to assess the safety and efficacy of TPVLC with PLD.

Material and methods: We screened patients with paravalvular leak (PVL) after surgical valve replacement (SVR). Heart failure symptoms and/or hemolytic anemia were indications for TPVLC. Patients were selected according to PVL anatomy by RT 3D TEE. Only those considered appropriate for closure with a single PLD were enrolled. The procedures were performed via transvascular or transapical access using type W (waist) PLDs only.

Results: Thirty patients with 34 PVLs (18 aortic, 16 mitral) were included. We implanted 35 PLDs with a total device success rate of $94.3 \%$ (100\% for aortic, $88.2 \%$ for mitral). The procedural success rate, encompassing device success without in-hospital complications, was $94.1 \%$ (100\% for aortic, $93.8 \%$ for mitral). During the follow-up period we recorded an increase of hemoglobin concentration (3.9 to $4.1 \mathrm{~g} / \mathrm{dl}$ ), red blood count (11.6 to $12.2 \mathrm{M} / \mathrm{mm}^{3}$ ) and functional improvement by NYHA class.

Conclusions: Paravalvular leak device type $\mathrm{W}$ is a promising TPVLC device, but meticulous preselection of patients based on imaging of PVL anatomy is a prerequisite. A PLD should only be chosen for channels shorter than $5 \mathrm{~mm}$. Size of the device should match the PVL cross-sectional area without any oversizing. Such an approach facilitates high device and procedural success rates.

Key words: percutaneous closure, paravalvular leak, occluder, prosthetic heart valve.

\section{Introduction}

Paravalvular leak (PVL) after surgical valve replacement (SVR) may occur in up to $10 \%$ of patients, and $2-3 \%$ of them will require repair due to heart failure (HF) or hemolytic anemia $[1,2]$. The transcatheter PVL closure (TPVLC) was introduced into clinical practice 20 years ago [3]. Transcatheter PVL closure, with proven beneficial effect on HF symptoms and hemolysis [4], has been recently granted a class Ila recommendation by the AHA/ACC [5]. Transcatheter PVL closure was initially performed as an "off-label" indication with ASD/VSD/PDA occluders and later with vascular embolization devices [6-9].

The first TPVLC-dedicated device to receive CE marking was the Paravalvular Leak Device (PLD; Occlutech
International $A B$, Sweden). It has a double-disc design and can have either a narrow or a wide central module (Figure 1). The discs, filled with fabric for improved sealing, are available in square or rectangular shape. The first TPVLC with PLD was reported in 2014 [10]. The present registry is intended to assess the safety and the efficacy of TPVLC using PLD performed after SVR.

\section{Material and methods}

Adult patients were scheduled for TPVLC when presenting with hemodynamically significant PVL at a surgically implanted prosthetic valve or a PVL causing hemolytic anemia with the need for at least one packed red blood cell (PRBC) transfusion. Hemodynamic significance

\section{Corresponding author:}

Grzegorz Smolka MD, PhD, FESC, 3 ${ }^{\text {rd }}$ Division of Cardiology, Medical University of Silesia, 45-47 Ziołowa St, 40-635 Katowice, Poland, phone: +48 501201622 , e-mail: grsm18@wp.pl

Received: 29.02.2016, accepted: 3.03.2016. 

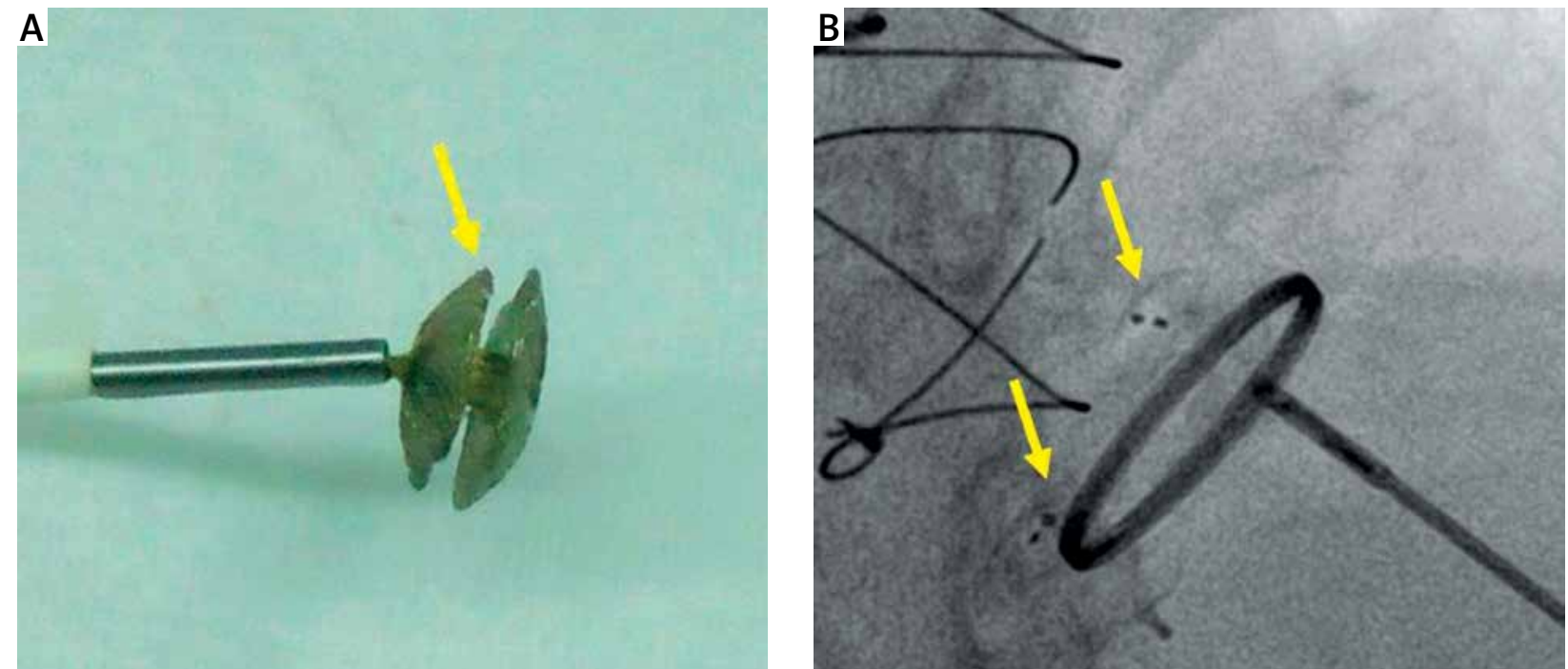

Figure 1. PLD type $\mathrm{W}$ - with wide middle module: A - device (arrow) connected to bioptome-like delivery wire, B - 2 PLDs (arrows) after transapical implantation into mitral PVLs

was defined by (a) HF symptoms (NYHA class II-IV) that could be attributed to paravalvular regurgitation despite optimal pharmacotherapy; (b) presence of $\geq 2+P V L$ jet in color Doppler (CD) mapping accompanied by at least one of the following indirect echocardiographic indicators of significant regurgitant flow:

- In case of mitral PVL: (1) systolic flow reversal in at least one of the pulmonary veins, (2) increased calculated pulmonary artery systolic pressure, (3) lack of left atrium (LA) size reduction after mitral valve replacement (MVR) or recurrent and progressive LA dilation in follow-up, (4) forward transprosthetic flow velocity higher than expected with given prosthesis type and size, provided normal function of prosthetic leaflets;

- In case of aortic PVL: (1) holodiastolic flow reversal in proximal part of descending aorta, (2) lack of left ventricle (LV) size reduction after aortic valve replacement (AVR) or recurrent and progressive LV dilation in postoperative course, and (3) forward transprosthetic flow velocity higher than expected with given prosthesis type and size, provided normal function of prosthetic leaflets.

Patients are disqualified from TPVLC in our institution if an indication for classical valve replacement surgery (prosthetic valve instability, need for coronary artery bypass grafting), active infective endocarditis (IE) or unexplained elevation of inflammatory markers (WBC, CRP) is found. In the current analysis, we included only patients treated with one version of PLD with the wide middle module (Waist type). The second available version (Twist) is also a double-disc device, but the middle module is a thin connector. The Waist-type device has self-centering properties, while the Twist type is more useful for narrow channels and multiple parallel narrow channels. Boty PLD types are available in square and rectangular shapes in several sizes.

We used either transseptal or transapical (with lateral mini-thoracotomy) access for mitral PVLs and retrograde transarterial access for aortic PVLS. Transvascular procedures were performed under conscious sedation, while transapical ones were performed under general anesthesia [11]. We implanted a single Waist type PLD into each PVL. The size of the device was chosen according to the dimensions of PVL vena contracta by RT 3D TEE. Accordingly, we matched width and length of the PLD middle module (waist) to the minimum (width) and maximum (length) dimensions of the PVL cross-sectional area (CSA) in multi-planar reconstruction - Figure 2.

We refrained from oversizing to avoid any folding of the device and thus to ensure full expansion of both discs. Additionally, we also measured the length of the channel in the plane perpendicular to the prosthesis' sewing ring (and CSA plane). This dimension reflected the expected distance separating PLD discs after implantation.

Since different TPVLC scenarios, such as repeated implantation during the same procedure as well as repeated procedure after failed implantation, are possible, the following definitions were outlined for this paper:

- Procedure: gaining access + PVL location and crossing + device implantation + delivery system removal + access site protection (suture(s)/closure device);

- Procedure time: from arterial/venous puncture for the transvascular approach or skin incision for the transapical approach until final hemostasis;

- Device implantation: crossing PVL channel with delivery sheath + occluder introduction through delivery sheath + occluder implantation into PVL channel + delivery cable release.

Study endpoints:

- Device success: device implantation with stable device position without interference with prosthetic valve discs/leaflets and with PVL CSA reduction by at least 90\%; calculated per number of attempted implantations; 


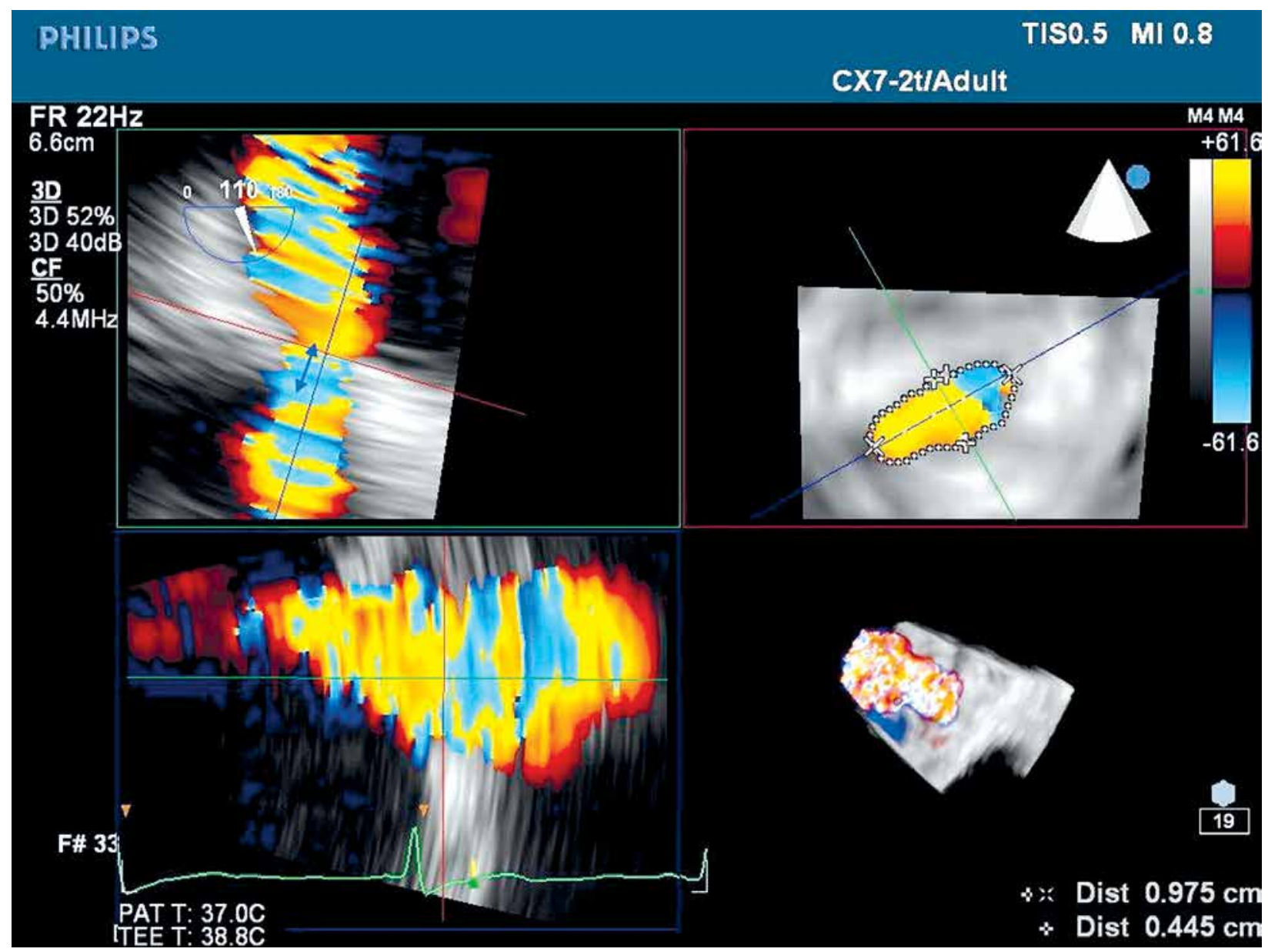

Figure 2. Multi-planar reconstruction of RT 3D TEE data set - measurement of PVL channel length (left panel, blue arrow) and cross sectional area (right panel) of mitral PVL

- Procedural success: device success + no safety endpoints during hospitalization; procedural success rate was calculated per number of attempted procedures;

- Safety endpoints (in-hospital and after 30 days of follow-up): TPVLC-related complications were modified VARC-2 [12] procedure-related complications, additionally including: prosthetic valve disc impingement occurring after plug deployment, PLD embolization, significant exacerbation of hemolysis defined as either procedure-induced hemolytic anemia requiring transfusion of at least 2 PRBC units (once the bleeding-related anemia was excluded) or clinically overt jaundice, and other complications as defined in VARC-2;

- NYHA class changes over 30 days of follow-up.

\section{Statistical analysis}

Changes were presented as mean and SD values for normal distribution, median and IQ values for non-normal distribution; all calculations were performed with commercially available software (MedCalc v.14.12.0).

The study was approved by the Ethics Committee of the Medical University of Silesia and supported by the
STRATEGMED II grant (National Centre for Research and Development, STRATEGMED2/269488/7/NCBR/2015).

\section{Results}

Thirty patients presenting with a total of 34 PVLs were included and followed up. Demographic and clinical data are listed in Table I. We performed 18 TPVLC procedures in the aortic location (including 2 PVLs in the same patient closed sequentially during two procedures) and 16 TPVLC procedures in the mitral location (including 2 PVLS in the same patient closed simultaneously via transapical access and a repeated procedure after failure of the first attempt in another patient). The study flowchart is shown in Figure 3.

The mitral location of the PVL was associated with longer procedural time, a higher dose of radiation (Table II) and an considerably lower procedural success rate. In general, such procedures required transseptal puncture, the creation of a loop in the left atrium using the telescopic catheter system. Also, the deployment of the device was more challenging in mitral PVL.

Procedural success was achieved in 32 (94.1\%) procedures, more frequently in aortic PVL $(18 ; 100 \%)$ than 
in mitral PVL $(15 / 17 ; 88.2 \%)$ cases. There was 1 case of device failure with exacerbation of hemolytic anemia. Table III shows the technical procedural aspects of PLD implantation.

The correlation between the sizes of implanted PLDs and PVLs' CSA dimensions by RT 3D TEE is presented in Figure 4. Influence of device oversizing on the effect (incomplete vs. total closure) was analyzed separately for the length and the width of PLD - see Figure 5 (incomplete closures are marked with filled circles, total closures with empty ones).

Residual paravalvular flow more often occurred in cases of device oversizing with regards to the length of PVL's CSA, but the correlation did not reach statistical significance $(p=0.068)$.

There were no major adverse cardiac and cerebrovascular event (MACCE) during hospitalization and no urgent surgical interventions. In 2 patients small access site pseudoaneurysms developed. They did not fulfill the VARC criteria and were successfully treated by thrombin injection. In one patient there was worsening of hemolytic anemia with jaundice, increase of bilirubin level (from $1.91 \mathrm{mg} / \mathrm{dl}$ to $3.2 \mathrm{mg} / \mathrm{dl}$ ) and lactate dehydroge-
Table I. Demographic and clinical data

\begin{tabular}{lc} 
Parameter & \multicolumn{1}{c}{ Result } \\
\hline Number of patients & 30 \\
\hline Age [years] & 63 (IQ 59-70) \\
\hline Female, $n$ (\%) & $19(63)$ \\
\hline Mitral PVL location & 13 \\
\hline Aortic PVL location & 17 \\
\hline NYHA on presentation, $n(\%):$ & 0 \\
\hline I & $7(23.3)$ \\
\hline II & $16(53.3)$ \\
\hline III & $7(23.3)$ \\
\hline IV & $4(13.2)$ \\
\hline Transfusion - dependent hemolytic anemia, $n$ (\%) & 11.6 (IQ 10.6-13.4) \\
\hline HGB [g\%] & $3.9($ (IQ 3.7-4.2) \\
\hline RBC [M/mm $\left.{ }^{3}\right]$ & $51.0($ IQ 36.0-57.2) \\
\hline LVEF [\%] & $7.3($ (IQ 4.1-11.3)
\end{tabular}

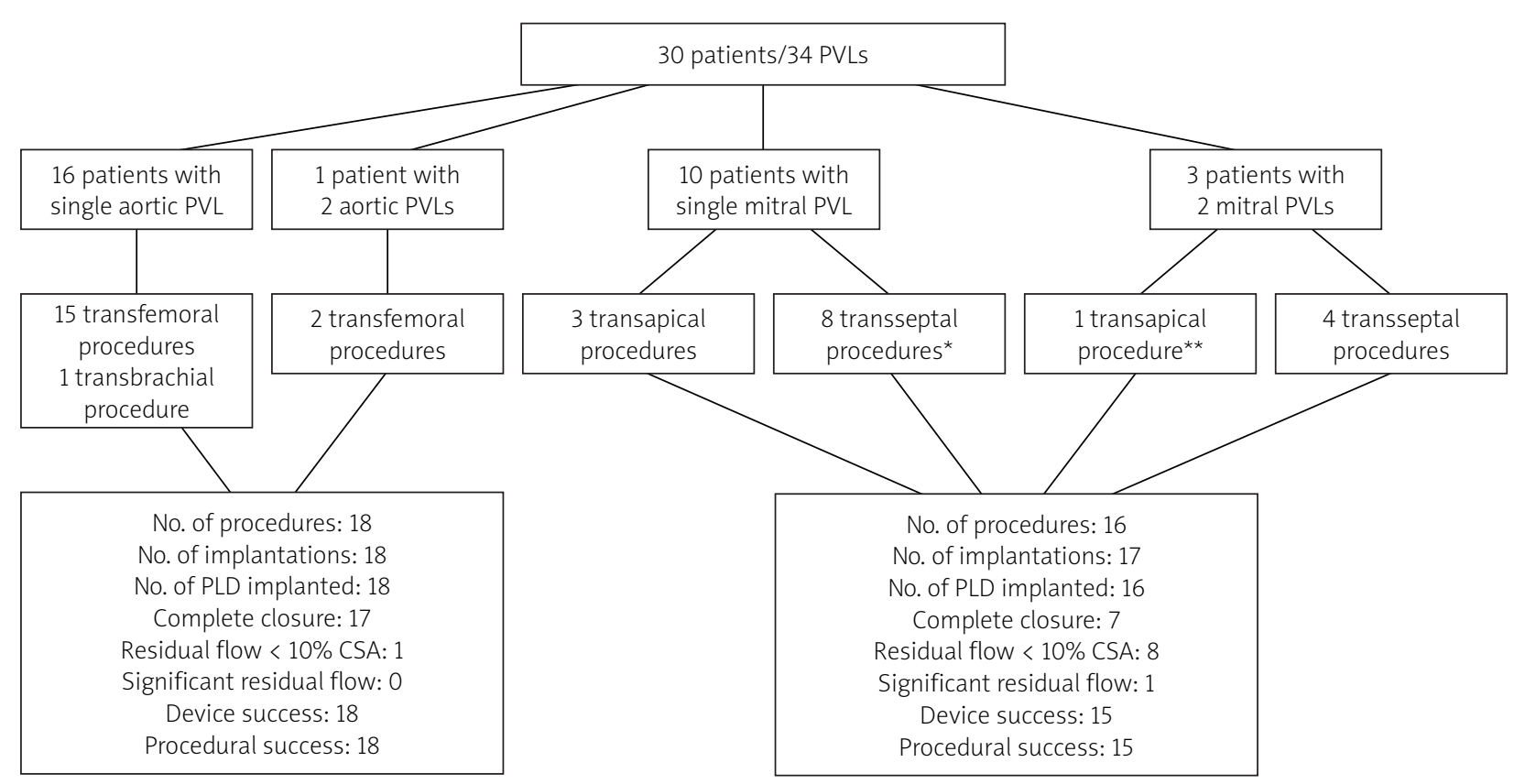

${ }^{*} 1 \mathrm{~s}$ attempt after failed implantation, ${ }^{* *}$ two $P L V$ s addressed in one procedure.

Figure 3. Study flowchart

Table II. Procedural data

\begin{tabular}{|c|c|c|}
\hline Parameter & Aortic PVL & Mitral PVL \\
\hline Procedure time [min] & $\begin{array}{l}\text { median 90, IQR 70-100 } \\
\quad(\min .25, \max .145)\end{array}$ & $\begin{array}{l}\text { median 125, IQR 95-180 } \\
\quad(\min .50, \text { max. 200) }\end{array}$ \\
\hline Radiation dose [mGy] & $\begin{array}{l}\text { median 732.5, IQR 383-1035.5 } \\
\text { (min. 195, max. 1937) }\end{array}$ & $\begin{array}{l}\text { median 980, IQ 541-1808 } \\
\text { (min. 267, max. 2220) }\end{array}$ \\
\hline Contrast medium volume $[\mathrm{ml}]$ & $\begin{array}{c}\text { median 50, IQ 40-67.5 } \\
(\min .20, \text { max. 280) }\end{array}$ & $\begin{array}{c}\text { used only in } 5 \text { cases } \\
\text { (20 } \mathrm{ml} \text { in } 4,40 \mathrm{ml} \text { in } 1 \text { patient) }\end{array}$ \\
\hline
\end{tabular}


Table III. Technical description of individual implantation steps

\begin{tabular}{|c|c|c|c|c|c|}
\hline $\begin{array}{l}\text { PVL location/prosthesis } \\
\text { mechanical ( } m) \\
\text { or biological }(b)\end{array}$ & $\begin{array}{l}\text { Delivery } \\
\text { sheath }\end{array}$ & $\begin{array}{l}\text { PLD } \\
\text { size }\end{array}$ & $\begin{array}{l}\text { PLD implanted into } \\
\text { PVL channel/optimal } \\
\text { rotation gained }\end{array}$ & $\begin{array}{l}\text { Release from } \\
\text { delivery cable }\end{array}$ & Device success \\
\hline Aortic/m & $8 \mathrm{Fr}^{\mathrm{a}}>7 \mathrm{Fr}^{\mathrm{b}, \mathrm{h}}$ & $10 \times 4$ & $\mathrm{Y} / \mathrm{Y}$ & Uneventful & Y (complete closure) \\
\hline Mitral/m & $9 \mathrm{Fr}^{\mathrm{e}}$ & $8 \times 4$ & $\mathrm{Y} / \mathrm{N}^{\mathrm{k}}$ & Uneventful & N (significant residual flow) \\
\hline Mitral/m & $12 \mathrm{Fr}^{\mathrm{f}}$ & $12 \times 5$ & $Y / Y$ & Uneventful & Y (mild residual leak) \\
\hline Aortic/b & $7 \mathrm{Fr}^{\mathrm{b}}$ & $8 \times 4$ & $Y / Y$ & Uneventful & Y (complete closure) \\
\hline Aortic/m & $6 \mathrm{Fr}^{\mathrm{c}}>6 \mathrm{Frc}^{\mathrm{ci}}$ & $6 \times 3$ & $Y / Y$ & $\begin{array}{l}\text { Repeated maneuvers } \\
\text { necessary }\end{array}$ & Y (mild residual leak) \\
\hline Aortic/m & $6 \mathrm{Fr}^{\mathrm{c}}$ & $6 \times 3$ & $\mathrm{Y} / \mathrm{N}^{\prime}$ & Uneventful & Y (complete closure) \\
\hline Aortic/b & $7 \mathrm{Fr}^{\mathrm{b}}$ & $8 \times 4$ & $Y / Y$ & Uneventful & Y (complete closure) \\
\hline Aortic/b & $7 \mathrm{Fr}^{\mathrm{b}}$ & $8 \times 4$ & $\mathrm{Y} / \mathrm{Y}$ & Uneventful & Y (complete closure) \\
\hline Aortic/m & $6 \mathrm{Fr}^{\mathrm{c}}$ & $6 \times 3$ & $Y / Y$ & Uneventful & Y (complete closure) \\
\hline Aortic/m & $6 \mathrm{Fr}^{\mathrm{c}}$ & $6 \times 3$ & $\mathrm{Y} / \mathrm{N}^{\prime}$ & Uneventful & Y (complete closure) \\
\hline Aortic/m & $7 \mathrm{Fr}^{\mathrm{b}}$ & $8 \times 4$ & $Y / Y$ & Uneventful & Y (complete closure) \\
\hline Mitral/m & $12 \mathrm{Fr}^{\mathrm{g}}$ & $12 \times 5$ & $Y / Y$ & Uneventful & Y (complete closure) \\
\hline Mitral/m & $9 \mathrm{Fr}^{\mathrm{e}}>12 \mathrm{Frgj}$ & $10 \times 4$ & $\mathrm{Y} / \mathrm{Y}$ & Uneventful & Y (mild residual leak) \\
\hline Mitral/b & $8 \mathrm{Frg}$ & $6 \times 3$ & $Y / Y$ & Uneventful & Y (mild residual leak) \\
\hline Mitral/b & $12 \mathrm{Fr}^{\mathrm{f}}$ & $12 \times 5$ & $\mathrm{Y} / \mathrm{Y}$ & Uneventful & N (significant residual flow) \\
\hline Mitral/m & $9 \mathrm{Fr}^{\mathrm{e}}$ & $8 \times 4$ & $\mathrm{Y} / \mathrm{N}^{\prime}$ & Uneventful & Y (complete closure) \\
\hline Aortic/b & $6 \mathrm{Fr}^{\mathrm{c}}$ & $4 \times 2$ & $Y / Y$ & $\begin{array}{l}\text { Repeated maneuvers } \\
\text { necessary }\end{array}$ & Y (complete closure) \\
\hline Mitral/m & $12 \mathrm{Fr}^{\mathrm{g}}$ & $12 \times 5$ & $Y / Y$ & Uneventful & Y (complete closure) \\
\hline Mitral/m & $12 \mathrm{Fr}^{\mathrm{f}}$ & $12 \times 5$ & $Y / Y$ & Uneventful & Y (mild residual leak) \\
\hline Aortic/b & $6 \mathrm{Fr}^{\mathrm{c}}$ & $4 \times 2$ & $\mathrm{Y} / \mathrm{N}^{\prime}$ & Uneventful & Y (complete closure) \\
\hline Aortic/m & $7 \mathrm{Fr}^{\mathrm{b}}$ & $8 \times 4$ & $Y / Y$ & Uneventful & Y (complete closure) \\
\hline Mitral/m & $9 \mathrm{Fr}^{\mathrm{e}}$ & $12 \times 5$ & $\mathrm{Y} / \mathrm{Y}$ & Uneventful & Y (mild residual leak) \\
\hline Mitral/m & $6 \mathrm{Fr}^{\mathrm{c}}$ & $6 \times 3$ & $Y / Y$ & Uneventful & Y (complete closure) \\
\hline Aortic/b & $6 \mathrm{Fr}^{\mathrm{c}}$ & $6 \times 3$ & $Y / Y$ & Uneventful & Y (complete closure) \\
\hline Aortic/b & $6 \mathrm{Fr}^{\mathrm{c}}$ & $6 \times 3$ & $Y / Y$ & Uneventful & Y (complete closure) \\
\hline Aortic/b & $6 \mathrm{Fr}^{\mathrm{c}}$ & $4 \times 2$ & $Y / Y$ & Uneventful & Y (complete closure) \\
\hline Aortic/m & $6 \mathrm{Fr}^{\mathrm{c}}$ & $6 \times 3$ & $\mathrm{Y} / \mathrm{N}^{\prime}$ & Uneventful & Y (complete closure) \\
\hline Mitral/m & $7 \mathrm{Fr}^{\mathrm{b}}$ & $8 \times 4$ & $Y / Y$ & Uneventful & Y (mild residual leak) \\
\hline Aortic/b & $8 \mathrm{Fr}^{\mathrm{a}, \mathrm{d}}$ & $12 \times 5$ & $Y / Y$ & $\begin{array}{l}\text { Repeated maneuvers } \\
\text { necessary }\end{array}$ & Y (complete closure) \\
\hline Mitral/m & $9 \mathrm{Fr}^{\mathrm{e}}$ & $8 \times 4$ & $Y / Y$ & Uneventful & Y (complete closure) \\
\hline Mitral/m & $6 \mathrm{Fr}^{\mathrm{c}}$ & $4 \times 2$ & $Y / Y$ & Uneventful & Y (complete closure) \\
\hline Aortic/m & $6 \mathrm{Fr}^{\mathrm{c}}$ & $6 \times 3$ & $Y / Y$ & Uneventful & Y (complete closure) \\
\hline Mitral/b & $12 \mathrm{Fr}^{\mathrm{f}}$ & $12 \times 5$ & $Y / Y$ & Uneventful & Y (complete closure) \\
\hline Mitral/m & $12 \mathrm{Fr}^{\mathrm{f}}$ & $12 \times 5$ & $N / Y$ & $n / a$ & N (occluder unstable, removed) \\
\hline Mitral/m & $13 \mathrm{Fr}^{\mathrm{f}}$ & $18 \times 10$ & $Y / Y$ & Uneventful & Y (mild residual leak) \\
\hline
\end{tabular}

aFlexor Shuttle Guiding Sheath (COOK Medical) $90 \mathrm{~cm}$, bFlexor Shuttle Guiding Sheath (COOK Medical) $110 \mathrm{~cm}$, cFlexor Shuttle Guiding Sheath (COOK Medical) $110 \mathrm{~cm},{ }^{d} 9 \mathrm{Fr}$ Amplatzer TorqVue too short for aortic location accessed from femoral puncture; undersized 8 Fr sheath $10 \mathrm{~cm}$ longer used, ${ }^{\circ}$ Amplatzer TorqVue (St Jude

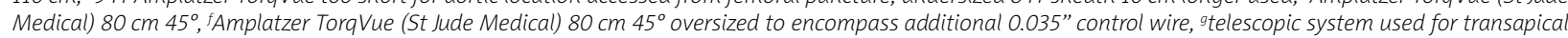
approach, consisting of a longer $(23 \mathrm{~cm})$ delivery sheath also used as a PLD loader and wider by $2 \mathrm{Fr}$. shorter $(15 \mathrm{~cm})$ sheath serving as a transapical access; both sheaths normally used for peripheral access, "is Fr Flexor Shuttle Guiding Sheath too short - exchanged for an undersized 7 Fr longer (110 cm) sheath, iDelivery sheath damaged by the occluder while passing the transversely located PVL channel; exchanged for a new one, the same type, 'delivery sheath damaged (kinked) in the left atrium; exchanged for a wider one, allowing simultaneous use of a stiff control wire, maintaining proper shape of the sheath inside the LA, krotation difficult due to severely angulated delivery sheath, 'rotation difficult due to the proximity of surrounding structures. 


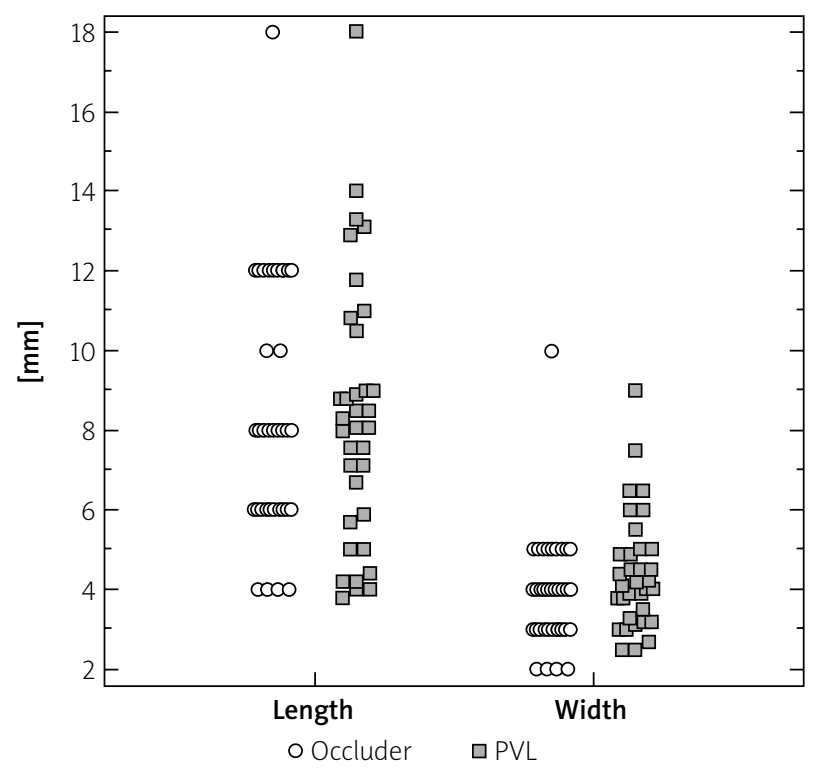

Figure 4. Correlation between the sizes of implanted PLDs and PVLs' dimensions (length and width of (SA) by RT 3D TEE

nase (LDH) activity (from $1214 \mathrm{IU} / \mathrm{I}$ to $2294 \mathrm{IU} / \mathrm{l}$ ). Both decreased spontaneously after 5 days to $1109 \mathrm{IU} / \mathrm{I}$ and $2.01 \mathrm{mg} / \mathrm{dl}$, respectively.

\section{Thirty-day follow-up}

No additional events or safety points were noted. None of the patients required transfusion, and the hemoglobin $(\mathrm{Hb})$ levels and RBC count increased in comparison to baseline preprocedural values ( $\mathrm{Hb}$ median of 4.1 (IQ 3.8-4.3) g\%, RBC 12.2 (IQ 10.8-13.3)).

Follow-up NYHA class was I in 14 (46.66\%) patients, class II in 13 (43.33\%) patients, and class III in $3(10 \%)$ patients at 1 month after TPVLC (in patients who underwent a repeated procedure after failure of the first attempt, NYHA class was assessed 30 days after the second one). The difference in NYHA class distribution at baseline and at the 30-day follow-up is presented in Figure 6.

\section{Discussion}

Direct comparison of our results with those reported for the so far most widely used AVP III occluder is difficult because of varying device/procedural success definitions. In one of the earlier papers [13], success, defined as implantation of the occluder, was achieved in $86 \%$. Later, Cruz-Gonzales et al. [7] achieved the procedural success of $90.9 \%$. It was semi-quantitatively defined as a reduction of paravalvular flow by at least one degree and might correspond to our device success. Another paper on the efficacy of multi-plug TPVLC technique using AVP III reported procedural success, demandingly defined as a reduction of regurgitation to not more than mild, of $76.5 \%$ for mitral and $100 \%$ for aortic PVLs [9]. Those results were similar to these currently achieved with PLD.

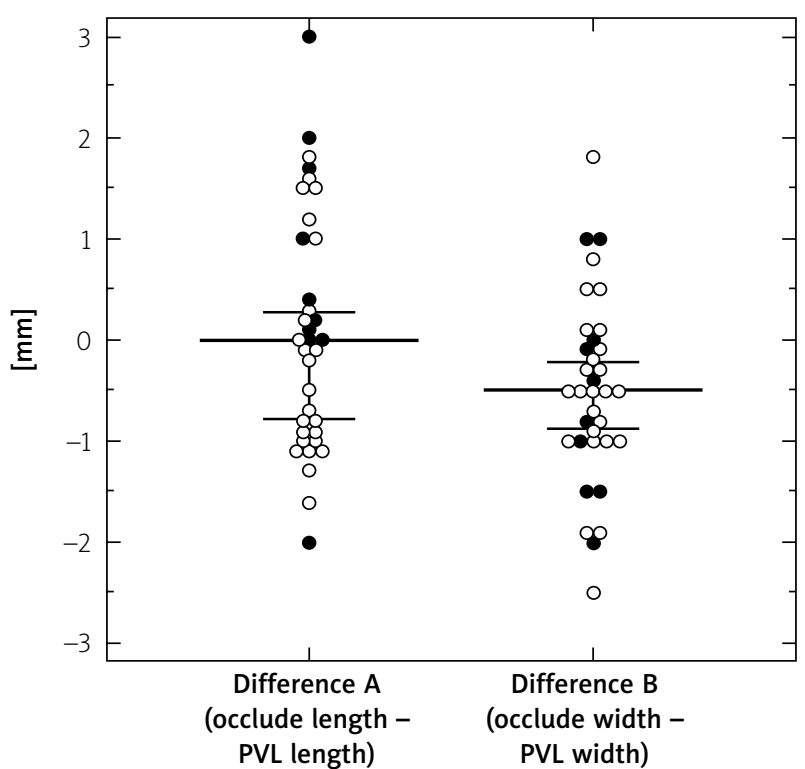

Figure 5. Influence of device oversizing on residual flow presence (filled circles) or absence (empty circles)

Our findings, however, may point to the superiority of PLD in patients carefully selected according to the PVL anatomy.

In the above papers, a multi-plug technique was needed for TPVLC with AVP III devices in the case of large PVLs. Even though efficient, such an approach is also somehow tricky as the increased number of occluding devices may result in their instability. Contrary to that, a PLD occluder facilitates closure of even large PVLs with a single device. Considering the substantial difference in PVL anatomy suitable for AVP or PLD, both techniques should probably be regarded as complementary rather than competitive, and a head-tohead comparison seems futile. A multi-plug AVP III approach seems reasonable for sealing long, irregularly shaped PVL channels. A single-plug PLD implantation appears superior in more regular, large PVLS with a short channel (shorter than the distance between discs, i.e. $5 \mathrm{~mm}$ ).

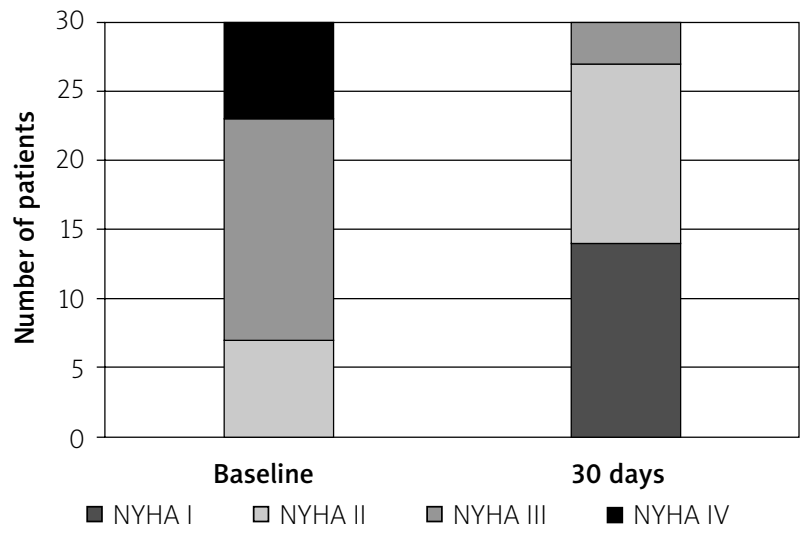

Figure 6. NYHA class distribution at baseline and at 30-day follow-up 
As shown in Table II, most PLD implantations required the use of delivery systems produced by other manufacturers. Even though such provisional solutions enabled the deployment in the majority of cases, we believe that development of a PLD-dedicated system could further improve the success rate. The current lack of such a system may also generate the need for transapical access in some mitral TPVLC cases.

As previously observed, the long-term clinical effect of TPVLC largely depends on the amount of residual flow [14]. Potential mechanisms of its occurrence after PLD implantation, besides the unlikely case of choosing a device with discs too small to cover the PVL, include:

- Occluder deformation caused by elongated PVL - if the length of the channel exceeds $4-5 \mathrm{~mm}$, the discs become spherical. It hinders the proper apposition of the fabric within them to surrounding tissue and excludes proper sealing.

- Squeezing of the waist causing similar disc deformation as in cases with a long channel - we observed residual leaks in patients in whom the size of the chosen waist device (PLD type W) even slightly exceeded the dimensions of the PVL.

Technical problems listed in Table II mostly resulted from a lack of TPVLC-dedicated delivery systems and were similar to those occurring with other types of occluders. No PLD-specific complications were identified. Apart from access site-related complications (the same delivery systems as with other occluders), we noted one case of significant post-procedural hemolytic anemia, the risk for which has been described since the beginning of TPVLC [3].

We carried out a pilot study assessing PLD procedural performance and safety/efficacy during short-term follow-up. The changes in anemia parameters and functional class should be interpreted cautiously, because of the small sample size, heterogeneity of the population and changes in medications over the course of follow-up. Long-term follow-up is necessary to conform the findings.

\section{Conclusions}

The Waist type of PLD appears to be a useful device for TPVLC in patients after SVR. Meticulous assessment of patients based on imaging of PVL anatomy is a prerequisite. Regardless of PVL CSA length and width, a PLD can be successfully used only for channels shorter than $5 \mathrm{~mm}$. When choosing the best size of the device, one should refrain from any attempts to oversize. In fact, we speculate that slight undersizing may be advisable to avoid any potential deformation of the occluding discs and ensure their proper apposition to surrounding tissue and full sealing. Such use of PLD facilitates high device and procedural success rates. Further elaboration of this technology should probably focus on developing dedicated transvascular delivery systems.

\section{Conflict of interest}

The authors declare no conflict of interest.

\section{References}

1. Davila-Roman VG, Waggoner AD, Kennard ED, et al. Prevalence and severity of paravalvular regurgitation in the Artificial Valve Endocarditis Reduction Trial (AVERT) echocardiography study. J Am Coll Cardiol 2004; 44: 1467-72.

2. Hwang HY, Choi JW, Kim HK, et al. Paravalvular leak after mitral valve replacement: 20-year follow-up. Ann Thorac Surg 2015; 100: 1347-52.

3. Hourihan M, Perry SB, Mandell VS, et al. Transcatheter umbrella closure of valvular and paravalvular leaks. J Am Coll Cardiol 1992; 20: 1371-7.

4. Millan X, Skaf S, Joseph L, et al. Transcatheter reduction of paravalvular leaks: a systematic review and meta-analysis. Can J Cardiol 2015; 31: 260-9.

5. Nishimura RA, Otto CM, Bonow RO, et al. 2014 AHA/ACC guideline for the management of patients with valvular heart disease: executive summary: a report of the American College of Cardiology/American Heart Association Task Force on Practice Guidelines. J Am Coll Cardiol 2014; 63: 2438-88.

6. Nietlispach F, Johnson M, Moss RR, et al. Transcatheter closure of paravalvular defects using a purpose-specific occluder. JACC Cardiovasc Interv 2010; 3: 759-65.

7. Cruz-Gonzalez I, Rama-Merchan JC, Arribas-Jimenez A, et al. Paravalvular leak closure with the Amplatzer Vascular Plug III device: immediate and short-term results. Rev Esp Cardiol (Engl Ed) 2014; 67: 608-14.

8. Fiszer R, Smolka G, Szkutnik M, Bialkowski J. Transcatheter aortic paravalvular leak closure using 3 Amplatzer Vascular Plug III devices in a child. Postep Kardiol Inter 2015; 11: 156-7.

9. Smolka G, Pysz P, Jasinski M, et al. Multiplug paravalvular leak closure using Amplatzer Vascular Plugs III: A prospective registry. Catheter Cardiovasc Interv 2016; 87: 478-87.

10. Goktekin O, Vatankulu MA, Tasal A, et al. Transcatheter trans-apical closure of paravalvular mitral and aortic leaks using a new device: first in man experience. Catheter Cardiovasc Interv 2014; 83: 308-14.

11. Smolka G, Pysz P, Jasinski M, et al. Transapical closure of mitral paravalvular leaks with use of amplatzer vascular plug III. J Invasive Cardiol 2013; 25: 497-501.

12. Kappetein AP, Head SJ, Genereux P, et al. Updated standardized endpoint definitions for transcatheter aortic valve implantation: the Valve Academic Research Consortium-2 consensus document. J Thorac Cardiovasc Surg 2013; 145: 6-23.

13. Ruiz CE, Jelnin V, Kronzon I, et al. Clinical outcomes in patients undergoing percutaneous closure of periprosthetic paravalvular leaks. J Am Coll Cardiol 2011; 58: 2210-7.

14. Sorajja P, Cabalka AK, Hagler DJ, Rihal CS. Long-term follow-up of percutaneous repair of paravalvular prosthetic regurgitation. J Am Coll Cardiol 2011; 58: 2218-24. 